Elsevier Editorial System(tm) for Nuclear

Inst. and Methods in Physics Research, A

Manuscript Draft

Manuscript Number: NIMA-D-18-01239

Title: Nuclear fragment identification with $\$ \backslash$ Delta\$E-E telescopes exploiting silicon carbide detectors

Article Type: Full length article

Section/Category: Gamma, X-ray and Charged Particle Detectors

Keywords: Silicon carbide detectors, nuclear fragment identification, $\$ \backslash D e l t a \$ E-E$ telescope, pulse shape analysis

Corresponding Author: Professor Gabriele Pasquali, PhD

Corresponding Author's Institution: University of Florence

First Author: Gabriele Pasquali, PhD

Order of Authors: Gabriele Pasquali, PhD; Caterina Ciampi; Carmen Altana; Maurizio Bini; Maurizio Boscardin; Lucia Calcagno; Giovanni Casini; Giuseppe Cirrone; Alberto Fazzi; Dario Giove; Giuseppe Gorini; Luca Labate; Francesco La Via; Gaetano Lanzalone; Grazia Litrico; Annamaria Muoio; Pietro Ottanelli; Giacomo Poggi; Sebastiana Puglia; Marica Rebai; Sabina Ronchin; Antonello Santangelo; Andrea A Stefanini; Antonio Trifirò; Salvo Tudisco; Massimo Zimbone

Abstract: A study of the response of three $\$ \backslash$ Delta\$E-E telescopes to fragments produced in nuclear interactions at $40 \$ \backslash$, \$AMeV is presented. All the employed telescopes feature silicon carbide (SiC) detectors for at least one detection stage. Two identification methods have been used and their performance discussed: the \$ $\backslash$ Delta\$E-E technique and the Pulse Shape Analysis technique (for identification of nuclear fragments stopped in a single sic layer). Identification capabilities similar to those obtained with the best available silicon detectors have been found for the Sic detector prototypes studied in this work. 
Dear Editor,

Please find enclosed one original of the manuscript titled:

"Nuclear fragment identification with $\Delta \mathrm{E}-\mathrm{E}$ telescopes exploiting silicon carbide detectors"

by the authors: Caterina Ciampi et al.

In the framework of the SiCILIA collaboration, we have tested a few p-n junction semiconductor detectors made of silicon carbide (SiC). The detectors have been used as detection stages in three $\Delta \mathrm{E}$-E telescopes for nuclear fragment identification.

The active area of the detectors is $1 \mathrm{~cm}^{2}$, which is large enough to build detector arrays covering a large solid angle with a reasonable number of detector pads. The interest of SiC as a detector material comes from its greater expected radiation hardness with respect to silicon, making $\mathrm{SiC}$ a promising candidate for detector arrays working with the future highluminosity low-energy heavy ion accelerators (like the upgraded Superconducting Cyclotron at LNS-Catania). In this paper we examine the performance of $\mathrm{SiC}$ based $\triangle \mathrm{E}$-E telescopes when used to identify nuclear fragments using both the $\Delta \mathrm{E}-\mathrm{E}$ technique and the Pulse Shape Analysis (PSA). To our knowledge, it is the first time that SiC detectors are characterized in that respect. We have obtained comforting results, showing that $\mathrm{SiC}$ detectors, even at the present prototype stage, can reach a performance comparable to the best available silicon detectors used for the same application, those of the FAZIA apparatus.

As corresponding author, I hereby declare that the manuscript is not under review for publication elsewhere and that the data have not been published previously. All co-authors have read the manuscript and agree to its submission. Hoping the paper will satisfy the requirements of the Journal I send you my Best Regards.

Yours Sincerily,

Prof. Gabriele Pasquali

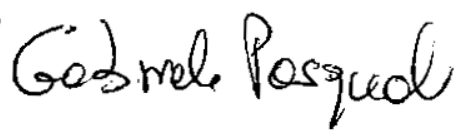

Dipartimento di Fisica e Astronomia dell'Università degli Studi di Firenze

and INFN-Sezione di Firenze,

via G. Sansone 1, 50019 Sesto Fiorentino (FI), Italia

Tel.: +39-05504572253

e-mail address: pasquali@fi.infn.it 


\title{
Nuclear fragment identification with $\Delta$ E-E telescopes exploiting silicon carbide detectors
}

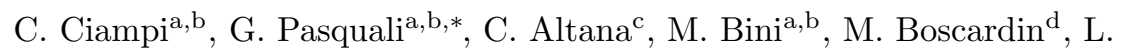 \\ Calcagno $^{\mathrm{e}, \mathrm{f}}$, G. Casini ${ }^{\mathrm{b}}$, G.A.P. Cirrone ${ }^{\mathrm{c}}$, A. Fazzi ${ }^{\mathrm{g}, \mathrm{h}}$, D. Giove ${ }^{\mathrm{g}, \mathrm{h}}$, G. Gorini ${ }^{\mathrm{i}, \mathrm{j}}$, \\ L. Labate ${ }^{\mathrm{k}}$, F. La Via ${ }^{\mathrm{c}, \mathrm{l}}$, G. Lanzalone ${ }^{\mathrm{c}, \mathrm{m}}$, G. Litrico ${ }^{\mathrm{n}}$, A. Muoio ${ }^{\mathrm{c}}$, P.

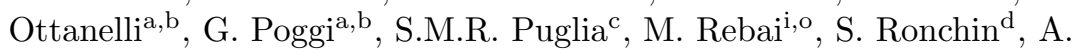

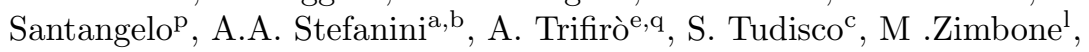 \\ for the SiCILIA collaboration \\ ${ }^{a}$ Dipartimento di Fisica e Astronomia, Università di Firenze, via G.Sansone 1, 50019 Sesto \\ Fiorentino (FI), Italy \\ ${ }^{b}$ INFN-Firenze, via G.Sansone 1, 50019 Sesto Fiorentino (FI), Italy \\ ${ }^{c}$ INFN-LNS, Via S.Sofia 62, 95123 Catania, Italy \\ ${ }^{d}$ TIFPA-INFN and FBK-Trento, via Sommarive 14, 38123 Povo Trento, Italy \\ ${ }^{e}$ INFN-Catania, Via S.Sofia 64 Catania, Italy \\ ${ }^{f}$ Department of Physics and Astronomy, University of Catania, Via S.Sofia 64 Catania, \\ Italy \\ ${ }^{g}$ INFN-Milano, via Celoria 16, 20133 Milano, Italy \\ ${ }^{h}$ Dept. of Energy, Politecnico di Milano, via Celoria 16, 20133 Milano, Italy \\ ${ }^{i}$ INFN-Milano Bicocca, Piazza della Scienza 3, 20126 Milano, Italy \\ ${ }^{j}$ Phys. Depart. of Bicocca University, Piazza della Scienza 3, 20126 Milano, Italy \\ ${ }^{k}$ INO-CNR, via G. Moruzzi 1, 56124 Pisa, Italy \\ ${ }^{l} I M M-C N R$, VIII Strada, 5, 95121 Catania, Italy \\ ${ }^{m}$ Facoltà di Ingegneria e Architettura, Università Kore, Cittadella Universitaria, 94100 \\ Enna, Italy \\ ${ }^{n}$ LPE, XVI Strada, 95121, Catania, Italy \\ ${ }^{o}$ IFP-CNR Via Cozzi 53, 20125 Milano \\ ${ }^{p}$ STMicroelectronics, Stradale Primosole, 50, 95121 Catania, Italy \\ ${ }^{q}$ Dipartimento di Scienze MIFT dell'Università di Messina, v.le F. S. D'Alcontres 31, \\ 98166 Messina, Italy
}

\begin{abstract}
A study of the response of three $\Delta \mathrm{E}-\mathrm{E}$ telescopes to fragments produced in nuclear interactions at $40 \mathrm{AMeV}$ is presented. All the employed telescopes feature silicon carbide $(\mathrm{SiC})$ detectors for at least one detection stage. Two identification methods have been used and their performance discussed: the $\Delta \mathrm{E}-\mathrm{E}$ technique and the Pulse Shape Analysis technique (for identification of nuclear fragments stopped in a single $\mathrm{SiC}$ layer). Identification capabilities similar to
\end{abstract}

\footnotetext{
* Corresponding author

Email address: pasquali@fi.infn.it (G. Pasquali)
} 
those obtained with the best available silicon detectors have been found for the $\mathrm{SiC}$ detector prototypes studied in this work.

Keywords: Silicon carbide detectors, nuclear fragment identification, $\Delta \mathrm{E}-\mathrm{E}$ telescope, pulse shape analysis

2010 MSC: 00-01, 99-00

\section{Introduction}

The use of silicon detectors as high resolution charged particle detectors is widespread in nuclear physics [1 5]. The recently developed FAZIA array 6 has obtained unprecedented performances in terms of isotopic identification of nuclear fragments with silicon-based detector telescopes [7-10].

A challenge for the next generation of detector arrays is their use with high intensity beams. In fact, new experiments are being planned exploiting high beam intensities in order to study very rare events or measure very low cross sections, e.g. NUMEN [11. However, the performance of silicon detectors rapidly deteriorates for increasing fluence, especially when pulse shape stability with time is desired [12]. Frequent substitution of the silicon detectors is not always a viable solution because of its technical complexity and high cost. Therefore, charged particle detectors featuring both high radiation hardness and high resolution are needed in order to replace silicon detectors. Projects which could benefit from the availability of radiation-hard semiconductor detectors are, e.g., NuReLP [13], ELIMED [14] and FAZIA [6]. They all require radiation-hard detectors with excellent performance in terms of stability, energy resolution, timing and insensitivity to the visible light. Some of them also require a relative large detection area, e.g. larger than $1 \mathrm{~cm}^{2}$, with thicknesses in the range from $50 \mu \mathrm{m}$ to $1000 \mu \mathrm{m}$. The presence of dead layers should be avoided in order to allow for the implementation of stacked detector systems as $\Delta \mathrm{E}-\mathrm{E}$ telescopes.

In view of the potential application of silicon carbide $(\mathrm{SiC})$ as a radiationhard material, the SiCILIA collaboration (Silicon Carbide detectors for Intense Luminosity Investigations and Applications) has started [15]. During the last 
two years, SiCILIA has produced a few $\mathrm{SiC}$ detector prototypes featuring active layers either $10 \mu \mathrm{m}$ or $100 \mu \mathrm{m}$ thick. Their active area is either $0.5 \times 0.5 \mathrm{~cm}^{2}$ or $1 \times 1 \mathrm{~cm}^{2}$. For the present study, some of them were mounted in telescope configuration and used to detect nuclear fragments, in order to test their identification capabilities both with the $\Delta \mathrm{E}-\mathrm{E}$ and the Pulse Shape Analysis (PSA) 30 techniques.

The $\Delta \mathrm{E}$-E technique can be used for particles with kinetic energy large enough to pass through the first detector and low enough to be stopped in the second. The partition of the energy between the two detectors is different for different particles, due to their different stopping power [16]. When particles covering a large energy range must be detected, it is common practice to use telescopes with more than two elements. In the present work, a thick $\mathrm{CsI}(\mathrm{Tl})$ was used as third element, due to its large stopping power. In PSA, only particles stopped in the first detector (i.e. those that cannot be identified with the $\Delta \mathrm{E}$ E technique) are considered. In fact, different nuclear fragments having the 40 same kinetic energy have a different energy deposition profile as a function of the penetration depth, thus producing different charge collection times and, consequently, different pulse shapes [17 20$]$.

The aim of the experiment reported in this paper is to verify the performance of the first prototypes produced by SiCILIA, by detecting and identifying heavy 45 nuclear fragments produced over a large range of charges, masses and energies, like the one we expect in an actual physics experiment. To our knowledge, this is the first time that $\mathrm{SiC}$ detectors are used for this application. The results presented in this paper show that the achieved identification capabilities almost reach those obtained from the best available silicon detectors as, e.g., those of the FAZIA apparatus.

The paper is organized as follows. Section 2 contains details about the instrumentation, the detector properties and the method of analysis. Section 3 gives the results of the $\Delta \mathrm{E}-\mathrm{E}$ identification technique and, when relevant, a comparison with the intrinsic physical limit imposed by energy straggling; the energy calibration procedure is also illustrated. Section 4 presents the results 
Table 1: Structure of the employed telescopes. The reported thickness is the nominal value.

\begin{tabular}{cccc}
\hline Telescope & $\begin{array}{c}\text { 1st Stage } \\
\text { material (thickness) }\end{array}$ & $\begin{array}{c}\text { 2nd stage } \\
\text { material (thickness) }\end{array}$ & $\begin{array}{c}\text { 3rd stage } \\
\text { material (thickness) }\end{array}$ \\
\hline $\mathrm{A}$ & $\mathrm{SiC}(10 \mu \mathrm{m})$ & $\mathrm{SiC}(100 \mu \mathrm{m})$ & $\mathrm{CsI}(10 \mathrm{~cm})$ \\
$\mathrm{B}$ & $\mathrm{SiC}(100 \mu \mathrm{m})$ & $\mathrm{Si}(510 \mu \mathrm{m})$ & $\mathrm{CsI}(10 \mathrm{~cm})$ \\
$\mathrm{C}$ & $\mathrm{SiC}(100 \mu \mathrm{m})$ & $\mathrm{CsI}(10 \mathrm{~cm})$ & \\
\hline
\end{tabular}

of the PSA applied to a $100 \mu \mathrm{m}$ thick SiC detector. Conclusions are drawn in the last section.

\section{Experimental setup}

Our test was conducted in the Ciclope scattering chamber at the Laboratori

${ }_{60}$ Nazionali del Sud (LNS) in Catania. Beams of ${ }^{40} \mathrm{Ca}$ and ${ }^{48} \mathrm{Ca}$ at $40 \mathrm{AMeV}$ were used to produce nuclear collisions on a thin ${ }^{12} \mathrm{C}$ target. The choice of these particular reactions has been dictated by the physics experiment performed by the FAZIA apparatus in Ciclope at the same time. The grazing angles of these collisions in the laboratory frame are $0.7^{\circ}$ for ${ }^{40} \mathrm{Ca}$ and $0.6^{\circ}$ for ${ }^{48} \mathrm{Ca}$. Three different telescopes (see Table 1) were mounted in the scattering chamber, at distances of about $80 \mathrm{~cm}$ from the target. They were positioned at a polar angle of about $8^{\circ}$, i.e. as close to the grazing angle as allowed by the presence of the FAZIA apparatus. All telescopes exploit a $10 \mathrm{~cm}$ thick $\mathrm{CsI}(\mathrm{Tl})$ scintillator as last stage in order to stop even the most penetrating charged products. Two

70 telescopes (telescopes A and B) consisted of three stages: telescope A is a $\mathrm{SiC}-$ $\mathrm{SiC}-\mathrm{CsI}(\mathrm{Tl})$ telescope and telescope $\mathrm{B}$ is a $\mathrm{SiC}-\mathrm{Si}-\mathrm{CsI}(\mathrm{Tl})$ telescope (i.e. its second stage is a silicon detector). Telescope $\mathrm{C}$ is a two-stage $\mathrm{SiC}-\mathrm{CsI}(\mathrm{Tl})$ telescope.

In this paper, the different detectors will be identified by telescope, material and stage number: e.g., the second stage of telescope A is called A-SiC2. All semiconductor detectors except $\mathrm{A}-\mathrm{SiC} 2$ are reverse mounted, i.e. particles imp- 
ing on the low field side of the junction. In fact, it has been demonstrated that, for semiconductor junction detectors, the PSA gives its best performance when fragments imping on the low field side (rear side) 9, 20, 21 instead of the high field (front) side.

All $\mathrm{SiC}$ detectors employed in our test are p-n junction detectors produced from n-type $4 \mathrm{H}-\mathrm{SiC}$ 22]. They are either $10 \mu \mathrm{m}$ (A-SiC1) or $100 \mu \mathrm{m}$ thick (A-SiC2, B-SiC1 and C-SiC1), epitaxially grown on a highly doped $\mathrm{SiC}$ substrate [15. The substrate is $100 \mu \mathrm{m}$ thick for A-SiC1 and $300 \mu \mathrm{m}$ thick for the other $\mathrm{SiC}$ detectors. The doping of the active layer is in the range $0.3 \div$ $8 \times 10^{13} \mathrm{~cm}^{-3}$. The $\mathrm{p}+$ electrode (about $300 \mathrm{~nm}$ thick, doping in the range $10^{18} \div 10^{19} \mathrm{~cm}^{-3}$ ) is produced by epitaxy. A few edge structures, produced by ion implantation, are also present. The substrate is heavily doped so that the electric field within the substrate is virtually null. It must be noted that preserved in the epitaxy, so that particles entering the detector perpendicularly have a low probability of undergoing channeling, an undesired effect which can spoil the $\Delta \mathrm{E}-\mathrm{E}$ correlations [23]. Further details about the $\mathrm{SiC}$ detectors can be found in Ref. [15].

Table 2 reports the main features of the $\mathrm{SiC}$ detectors. The active area of the $\mathrm{A}-\mathrm{SiC} 1$ is $1 \times 1 \mathrm{~cm}^{2}$ and it is covered by a single electrode of the same size. The active area of the $100 \mu \mathrm{m}$ thick detectors is $1 \times 1 \mathrm{~cm}^{2}$ and it is divided into four square pads (of about $0.5 \times 0.5 \mathrm{~cm}^{2}$ area) by segmentation of the $\mathrm{p}+$ electrode. Except in one case, two or more pads have been connected in parallel to a single charge preamplifier for signal readout:

- the four pads of A-SiC2 have been connected to a single charge preamplifier;

- three of the four pads of the first stage of telescope B have been connected to a single charge preamplifier (in the following we will refer to them as B$\mathrm{SiC} 1)$; the fourth pad has been connected to a separate charge preamplifier and named B-SiC1bis, since its bias can reach a maximum of only $40 \mathrm{~V}$ 
Table 2: Main parameters of the $\mathrm{SiC}$ detectors. The reported thickness has been determined from the calibration procedure (see Sec. 3.1.

\begin{tabular}{cccccc}
\hline Detector & A-SiC1 & A-SiC2 & B-SiC1 & B-SiC1bis & C-SiC1a,b \\
\hline Thickness $(\mu \mathrm{m})$ & 13 & 100 & 100 & 100 & 100 \\
Dep. Voltage $(\mathrm{V})$ & 4 & $400-700$ & 25 & 25 & $400-700$ \\
Pads & 1 & 4 & 3 & 1 & 2 \\
Total Area $\left(\mathrm{cm}^{2}\right)$ & 1 & 1 & 0.75 & 0.25 & 0.5 \\
Substr. Thick. $(\mu \mathrm{m})$ & 100 & 300 & 300 & 300 & 300 \\
Mounting & rear & front & rear & rear & rear \\
Appl. Voltage $(\mathrm{V})$ & 50 & 150 & 150 & 40 & 150 \\
\hline
\end{tabular}

before breakdown occurs;

- the four pads of detector C-SiC1 have been connected in pairs to two separate charge preamplifiers: they have been named C-SiC1a and CSiC1b.

The silicon detector employed in telescope B (B-Si2) is $510 \mu \mathrm{m}$ thick with an active area of $2 \times 2 \mathrm{~cm}^{2}$ and a depletion voltage of about $290 \mathrm{~V}$. It has been produced by FBK (Trento, Italy) [24] and it is of the same type as those employed as second stage of the FAZIA telescopes [6].

The semiconductor detectors are all in transmission mounting, to avoid additional dead layers between the different active layers of the telescopes. The CsI(Tl) scintillators are of the same kind as those employed by the FAZIA apparatus [6]. They have an entrance surface of $2.05 \times 2.05 \mathrm{~cm}^{2}$ and are $10 \mathrm{~cm}$ thick. The scintillation light is read-out by a photodiode placed on the side opposite to the entrance side. The photodiode is read by a charge preamplifier.

Since the active area of the silicon detector and of the scintillators is larger than that of the $\mathrm{SiC}$ detectors, the $\mathrm{SiC}$ detectors are centered with respect to the $\mathrm{Si}$ and $\mathrm{CsI}(\mathrm{Tl})$ detectors and a $3 \mathrm{~mm}$ thick brass collimator $\left(0.9 \times 0.9 \mathrm{~cm}^{2}\right.$ area) is placed (centered) in front of each telescope in order to stop particles 

$\mathrm{SiC} 2$ and stopped in its substrate cannot be recognized and removed using the CsI information: they contribute to the intense oblique correlation visible in 


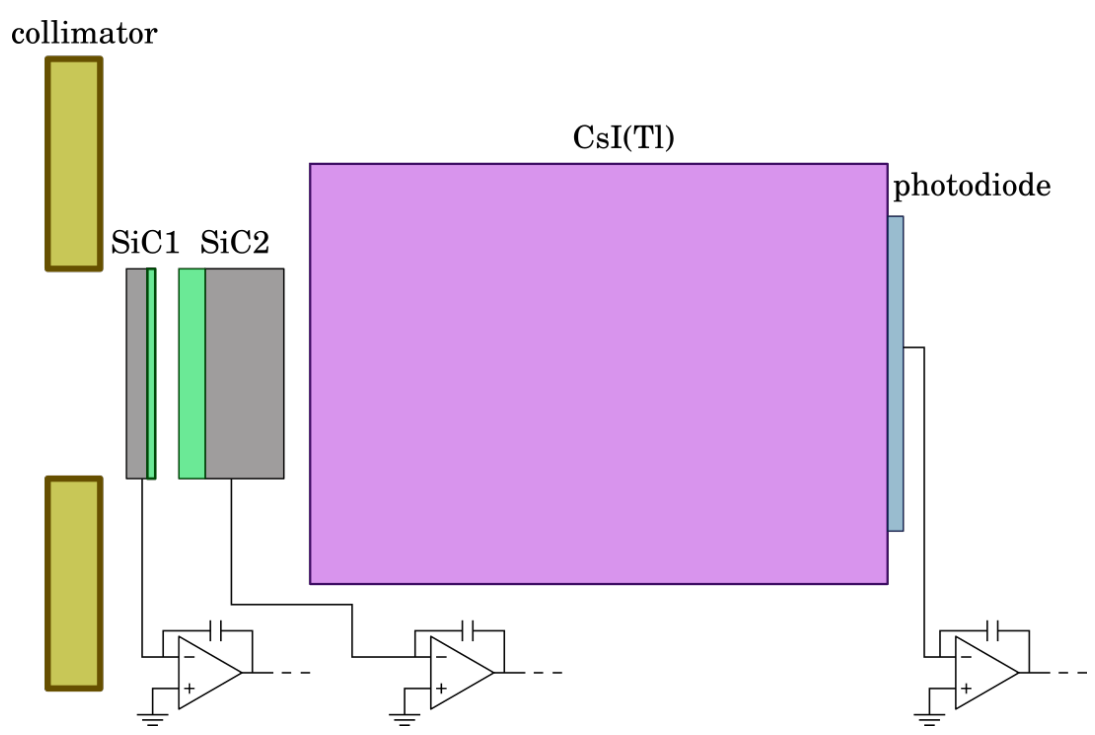

Figure 1: Sketch of telescope A, showing the SiC active areas (light green) together with their substrate (dark grey). The $0.9 \times 0.9 \mathrm{~cm}^{2}$ area collimator is also shown.

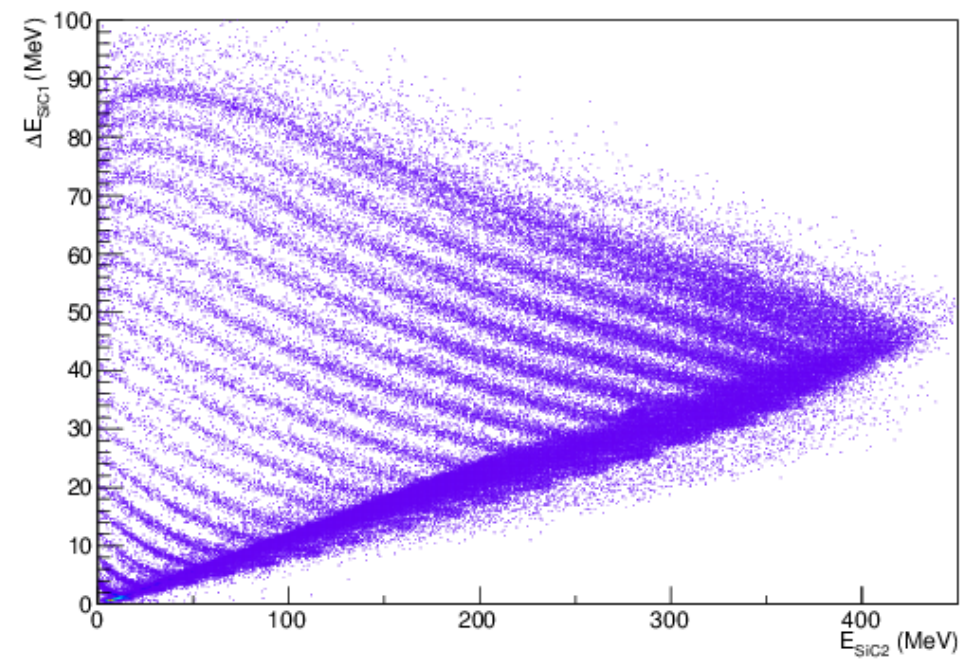

Figure 2: $\Delta \mathrm{E}-\mathrm{E}$ correlation obtained from the first two stages of telescope A (A-SiC1 and A-SiC2). 
Fig. 2. New prototypes having their substrate thickness reduced by lapping will be available for the future tests.

For the energy calibration of telescope A we exploit the so-called punchthrough points, i.e. the $(\mathrm{E}, \Delta \mathrm{E})$ coordinates of the rightmost end of each ridge in the $\Delta \mathrm{E}-\mathrm{E}$ correlation. This is the point for which the range of the particle is equal to the thickness of the $\mathrm{E}$ detector. The associated $\Delta \mathrm{E}$ and $\mathrm{E}$ values in $\mathrm{MeV}$ can be determined for a given element from energy loss calculations once the thickness of the active area is known for the two detectors 1

However, tests conducted with an alpha source indicated that the actual thickness of A-SiC1 could be greater than the nominal value of $10 \mu \mathrm{m}$. Therefore, in addition to the aforementioned method, two other methods have been used as a cross-check. In the first approach, the first stage ( $\mathrm{A}-\mathrm{SiC} 1)$ has been calibrated by using the leftmost end of each ridge, the point for which the range of the particle is equal to the thickness of the $\Delta \mathrm{E}$ detector. Then an $\mathrm{E}$ value has been determined for each ridge, for a point along the ridge at about half the $\Delta \mathrm{E}$ value of the punch-through in the first stage. The parameters entering into the calculation are the atomic and mass number of the fragment and the thickness of the $\Delta \mathrm{E}$ detector ${ }^{2}$. For this method, the thickness of the $\Delta \mathrm{E}$ detector is needed but not the one of the second stage. The second method exploits known amounts of charge injected in the preamplifier input by means of a precision pulser and a reference-capacitor. The pulser and reference-capacitor combination have been calibrated in energy both for silicon and $\mathrm{SiC}$, by comparison with the response of a silicon detector and a $\mathrm{SiC}$ detector to particles from a ${ }^{241} \mathrm{Am}$ source impinging on the junction side. The silicon detector and the $\mathrm{SiC}$ detector used for the comparison were of the same kind as those used in the experiment. The energy

\footnotetext{
${ }^{1}$ To better spot the punch-through points in the $\Delta$ E-E correlation of Fig. 2 a clean-up of the correlation, obtained by setting the minimum counts of the displayed bins to 2 , is needed.

${ }^{2}$ Whenever possible, the mass number to be used in the energy loss calculation has been determined from a weighted average based on the isotopic abundances measured by telescope B. In all other cases, the mass of the most abundant isotope of the given $\mathrm{Z}$ has been used. The same applies for the calibration method based on the punch-through points.
} 


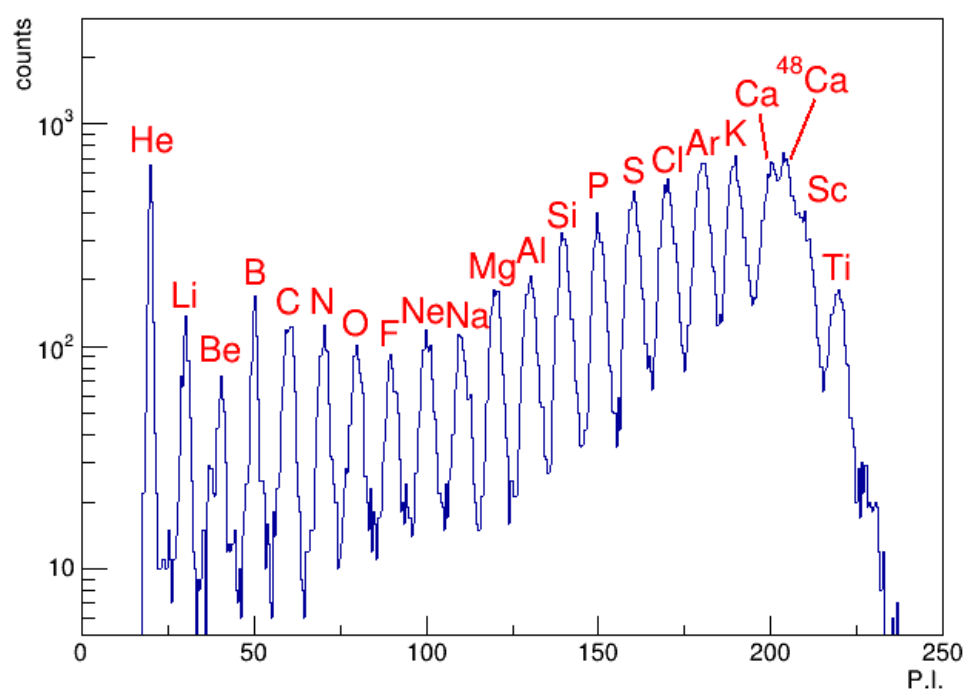

Figure 3: Particle Identification (PI) spectrum obtained from a linearization of the $\Delta \mathrm{E}-\mathrm{E}$ correlation shown in Fig. 2

lost in the source has been neglected. The energy lost entering the detector is estimated from the known thickness of the entrance dead layer. The relative uncertainty on the energy calibration of the pulser is estimated to be of the order of a few percent in the energy range of interest.

The three calibration methods agree to better than $1 \%$ provided that a value of $13 \mu \mathrm{m}$ is chosen for the $\Delta \mathrm{E}$ detector thickness, as reported in Tab. 2

In order to express quantitatively the $\mathrm{Z}$ resolution of the telescope, we apply a linearization procedure to the $\Delta \mathrm{E}-\mathrm{E}$ correlation as in Ref. [7]. The $\Delta \mathrm{E}-\mathrm{E}$ plot is linearized into a Particle Identification (PI) variable vs E plot, which is projected on the PI axis, giving the histogram shown in Fig. 3. Events falling on the oblique correlation due to punching through particles have been excluded from Fig. 3 by means of a graphical cut drawn on the correlation of Fig. 2 Hydrogen isotopes were not acquired due to the relatively high trigger threshold employed for telescope A.

The separation between adjacent elements can be quantified by means of a 
Table 3: Experimental and simulated (13 $\mu \mathrm{m}$ thick detector) FoM values obtained for the PI distributions in Fig. 3 The error on the reported FoM values is $\sim 10 \%$.

\begin{tabular}{lcc||lcc}
\hline Elements & FoM(exp.) & FoM(sim.) & Elements & FoM(exp.) & FoM(sim.) \\
\hline He-Li & 3.3 & 2.5 & Mg-Al & 1.2 & 1.9 \\
Li-Be & 2.3 & 2.2 & Al-Si & 1.1 & 1.8 \\
Be-B & 2.1 & 2.1 & Si-P & 1.1 & 1.7 \\
B-C & 1.7 & 2.3 & P-S & 1.1 & 1.6 \\
C-N & 1.4 & 2.2 & S-Cl & 1.0 & 1.6 \\
N-O & 1.2 & 2.6 & Cl-Ar & 1.0 & 1.5 \\
O-F & 1.2 & 2.6 & Ar-K & 0.9 & 1.0 \\
F-Ne & 1.2 & 2.2 & K-Ca & 0.9 & 1.1 \\
Ne-Na & 1.1 & 2.0 & Ca-Sc & 0.6 & 1.4 \\
Na-Mg & 1.2 & 1.9 & Sc-Ti & 0.8 & \\
\hline
\end{tabular}

Figure of Merit (FoM) 27]. Two peaks are conventionally considered well separated if their FoM is greater than 0.7, a value corresponding to a peak-to-valley ratio of $\sim 2$ for two Gaussian peaks having the same integral. Table 3 reports the FoM values obtained from the peaks of Fig. 3 (column FoM(exp.)) for the different elements pairs. Simulated $\Delta \mathrm{E}-\mathrm{E}$ correlations, including energy straggling effects, have been produced and subsequently analyzed in the same way as the experimental one, thus obtaining the FoM values for the different element pairs. The relative isotopic abundances in the simulation are adjusted to reproduce the experimental abundances measured by telescope B (see Sec. 3.2). The energy straggling and the electronic noise are the only resolution worsening effects included in the simulation. The energy straggling has been evaluated according to the semi-empirical parametrization of Yang [28] as done in [8. The electronic noise has been evaluated from the pulser events acquired during the experiment and amounts to about $200 \mathrm{keV}$ (in $\mathrm{SiC}$, full width at half maximum). A random value following a Gaussian distribution is added to the simulated energy values in such a way as to reproduce the correct amount of noise fluctuations. The 


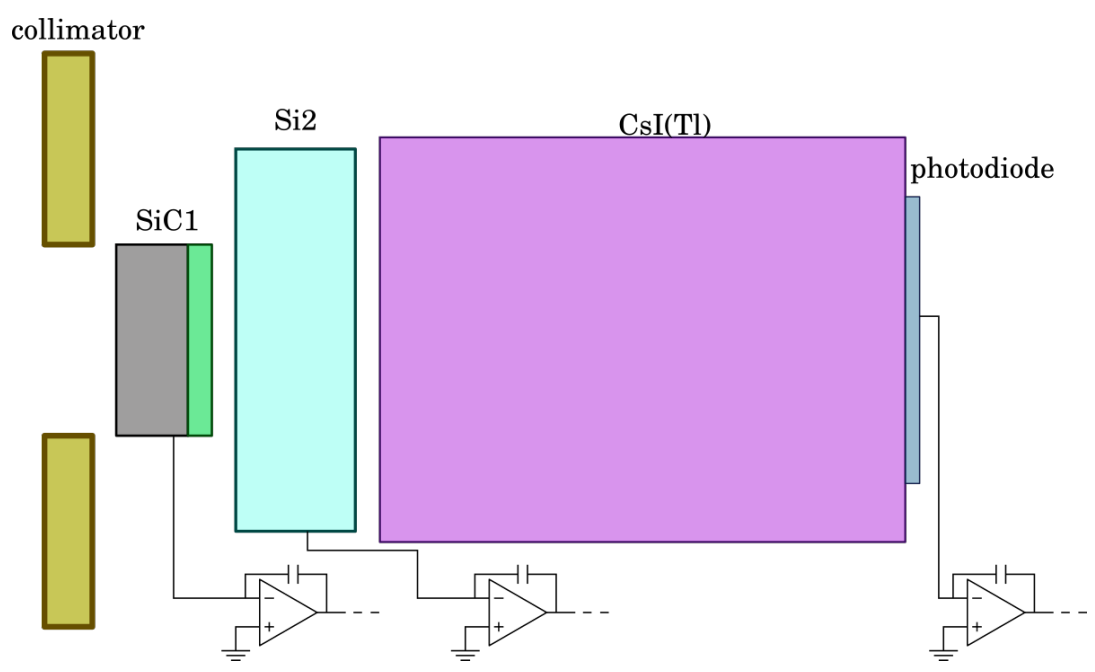

Figure 4: Sketch of telescope B. For the first element, the substrate (dark grey) and the active area (light green) are shown to scale. The picture is to scale separately for the vertical and horizontal directions.

results of the simulation are reported in column FoM(sim.) in Tab. 3 The FoM value for the lighter fragments is dominated by the electronic noise which prevents the separation of the different isotopes. For heavier fragments, some other effect could be at work, like a non uniformity of the active area thickness. The comparison shows that the intrinsic limit due to the energy straggling has not been reached. Further studies will be needed in order to disentangle the various contributions to the overall FoM.

\subsection{Telescope $B$}

Telescope B uses a $100 \mu \mathrm{m}$ thick $\Delta \mathrm{E} \mathrm{SiC} \mathrm{detector} \mathrm{(detector} \mathrm{B-SiC1).} \mathrm{The}$ second stage, B-Si2, is a $510 \mu \mathrm{m}$ thick silicon detector. As before, in order to avoid dead layers between the first and the second stage and also to optimize its PSA performance, $\mathrm{B}-\mathrm{SiC} 1$ is reverse mounted. A sketch of telescope $\mathrm{B}$ is shown in Fig. 4

A $\Delta \mathrm{E}-\mathrm{E}$ correlation between $\mathrm{B}-\mathrm{SiC} 2$ and $\mathrm{B}-\mathrm{Si} 2$ is shown in Fig. 5 . Element ridges up to $\mathrm{Z}=18$ are clearly separated (though statistics decreases for heavy ${ }_{225}$ fragments, beyond $\mathrm{Z}=16$, due to the reaction kinematics). The insets refer 


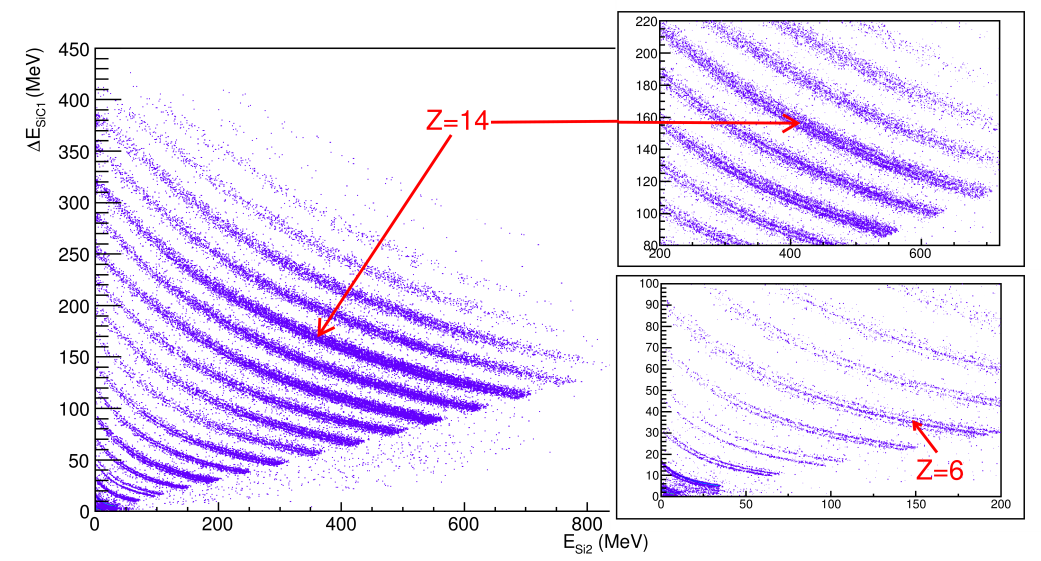

Figure 5: $\Delta \mathrm{E}-\mathrm{E}$ correlation obtained from the first two stages of telescope B (B-SiC1 and B$\mathrm{Si2}$ ). The top right inset shows that isotopic resolution is obtained up to silicon. The bottom right inset contains an expanded view of the Intermediate Mass Fragments region.

to the region around $\mathrm{Z}=14$, the maximum $\mathrm{Z}$ for which isotopic resolution is achieved. In Fig. 5, particles reaching the $\mathrm{CsI}(\mathrm{Tl})$ scintillator are vetoed by requiring the $\mathrm{CsI}(\mathrm{Tl})$ signal amplitude to be compatible with noise (three-sigma limit). Moreover, since the dead layer between $\mathrm{Si} 2$ and the $\mathrm{CsI}(\mathrm{Tl})$ is relatively thin (a few hundreds of nanometers), the amount of residual punching-through particles present in the $\Delta \mathrm{E}$ - $\mathrm{E}$ correlation is negligible. The better identification performance of telescope $\mathrm{B}$, with respect to telescope $\mathrm{A}$, is due to its thicker first stage, greatly reducing the uncertainty on $\Delta \mathrm{E}$ due to the effect of the energy straggling and of the thickness non uniformity. On the other hand, the energy threshold for atomic number identification via the $\Delta \mathrm{E}-\mathrm{E}$ technique is much lower for telescope A, due to its thinner first stage.

The correlation of Fig. 5 has been calibrated using two methods already employed for telescope A, namely the punch-through points method ${ }^{3}$ and the

\footnotetext{
${ }^{3}$ For telescope B, the calibration exploiting the punch-through points is also sensitive to the mass number (A) values assigned to the different isotopic ridges. If an ambiguity arises in assigning A to a given ridge or punch through point, the finally assigned value is the one which minimizes the $\chi^{2}$ value of the linear fit employed for the calibration.
} 
Table 4: Experimental FoM values obtained for the PI distributions in Fig. 6

\begin{tabular}{lc||lc||lc}
\hline Isotopes & FoM & Isotopes & FoM & Isotopes & FoM \\
\hline $\mathrm{p}-\mathrm{d}$ & 2.6 & ${ }^{12} \mathrm{C}-{ }^{13} \mathrm{C}$ & 1.0 & ${ }^{22} \mathrm{Na}-{ }^{23} \mathrm{Na}$ & 0.9 \\
$\mathrm{~d}-\mathrm{t}$ & 2.0 & ${ }^{14} \mathrm{~N}-{ }^{15} \mathrm{~N}$ & 0.9 & ${ }^{23} \mathrm{Na}-{ }^{24} \mathrm{Na}$ & 0.9 \\
${ }^{6} \mathrm{Li}-{ }^{7} \mathrm{Li}$ & 1.5 & ${ }^{15} \mathrm{O}-{ }^{16} \mathrm{O}$ & 1.0 & ${ }^{24} \mathrm{Mg}^{25} \mathrm{Mg}$ & 1.0 \\
${ }^{7} \mathrm{Li}-{ }^{8} \mathrm{Li}$ & 1.7 & ${ }^{16} \mathrm{O}-{ }^{17} \mathrm{O}$ & 0.8 & ${ }^{25} \mathrm{Mg}^{26} \mathrm{Mg}$ & 0.8 \\
${ }^{9} \mathrm{Be}-{ }^{10} \mathrm{Be}$ & 1.0 & ${ }^{18} \mathrm{~F}-{ }^{19} \mathrm{~F}$ & 0.9 & ${ }^{26} \mathrm{Al}-{ }^{27} \mathrm{Al}$ & 0.7 \\
${ }^{10} \mathrm{~B}-{ }^{11} \mathrm{~B}$ & 1.0 & ${ }^{20} \mathrm{Ne}-{ }^{21} \mathrm{Ne}$ & 0.8 & ${ }^{28} \mathrm{Si}^{29} \mathrm{Si}$ & $\mathbf{0 . 6}$ \\
${ }^{11} \mathrm{C}-{ }^{12} \mathrm{C}$ & 1.0 & ${ }^{21} \mathrm{Ne}-{ }^{22} \mathrm{Ne}$ & 0.8 & ${ }^{29} \mathrm{Si}^{30} \mathrm{Si}$ & $\mathbf{0 . 4}$ \\
\hline
\end{tabular}

calibrated pulser-capacitance combination. For both detectors (B-SiC1 and B$\mathrm{Si2}$ ), the two calibration methods agree within the estimated uncertainty when the nominal value is used for the detector thickness.

The correlation of Fig. 5 has been linearized by extracting a PI value for each event, as explained in Sec. 3.1. When the isotopic ridges are visible, the graphical lines used for linearization have been drawn for each isotope and assigned a PI value of $\mathrm{Z} \times 100+(\mathrm{A}-2 \mathrm{Z}) \times 10$. When isotopes are not resolved a value of $\mathrm{PI}=\mathrm{Z} \times 100$ is assigned. Figure 6 reports the PI histograms for different $\mathrm{Z}$ intervals, integrated over the whole energy range explored by the correlation of Fig. 5. The multiple-peak structures in panels (a)-(d), centered at integer multiples of 100 , correspond to fragments having the same $\mathrm{Z}$, each peak corresponding to a different isotope.

Table 4 reports the values of FoM calculated for a selection of adjacent isotopic peaks.

\subsection{Telescope $C$}

When a high stopping power is needed for the second stage of a telescope, a $\mathrm{CsI}(\mathrm{Tl})$ stage could be an alternative to a SiC-based one. In order to evaluate the performance of a $\mathrm{SiC}-\mathrm{CsI}(\mathrm{Tl})$ combination, a dedicate telescope has been prepared (telescope C). A sketch of telescope $\mathrm{C}$ is shown in Fig. 7. Its $\Delta \mathrm{E}$ stage 


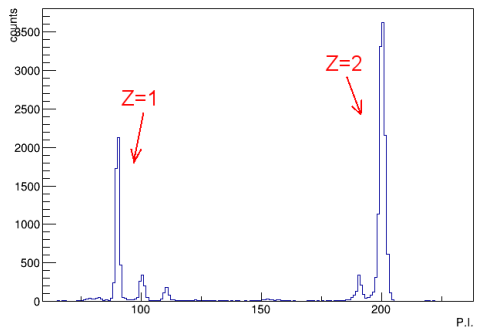

(a) $Z=1,2$

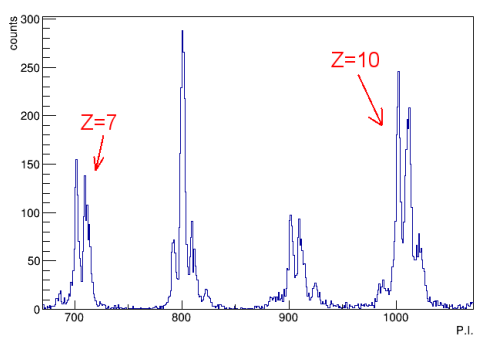

(c) $Z=7 \div 10$

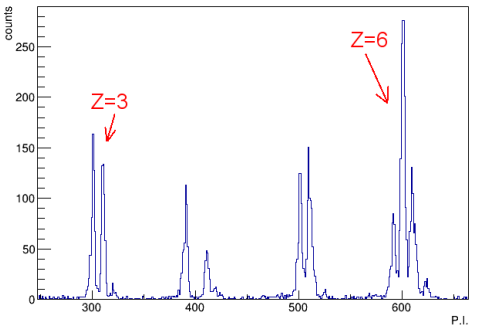

(b) $Z=3 \div 6$

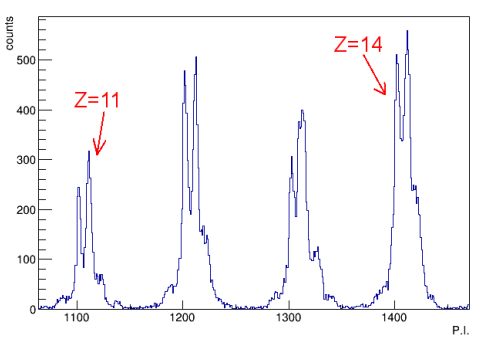

(d) $Z=11 \div 14$

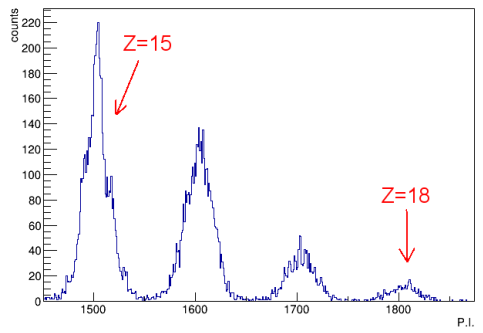

(e) $Z=15 \div 18$

Figure 6: PI histograms obtained from a linearization of the correlation shown in Fig. 5 . The multiple-peak structures centered at integer multiples of 100 correspond to fragments having the same $\mathrm{Z}$ (a few $\mathrm{Z}$ values are also indicated by the arrows). In panels (a)-(d) each peak corresponds to a different isotope. For $\mathrm{Z}>14$ isotopic resolution is not achieved, as it is evident from panel (e).

is a $100 \mu \mathrm{m}$ thick $\mathrm{SiC}$ detector having an active area of $1 \times 1 \mathrm{~cm}^{2}$ divided into four pads, grouped two by two in parallel for readout (see Sec. 2). From a C-V curve 


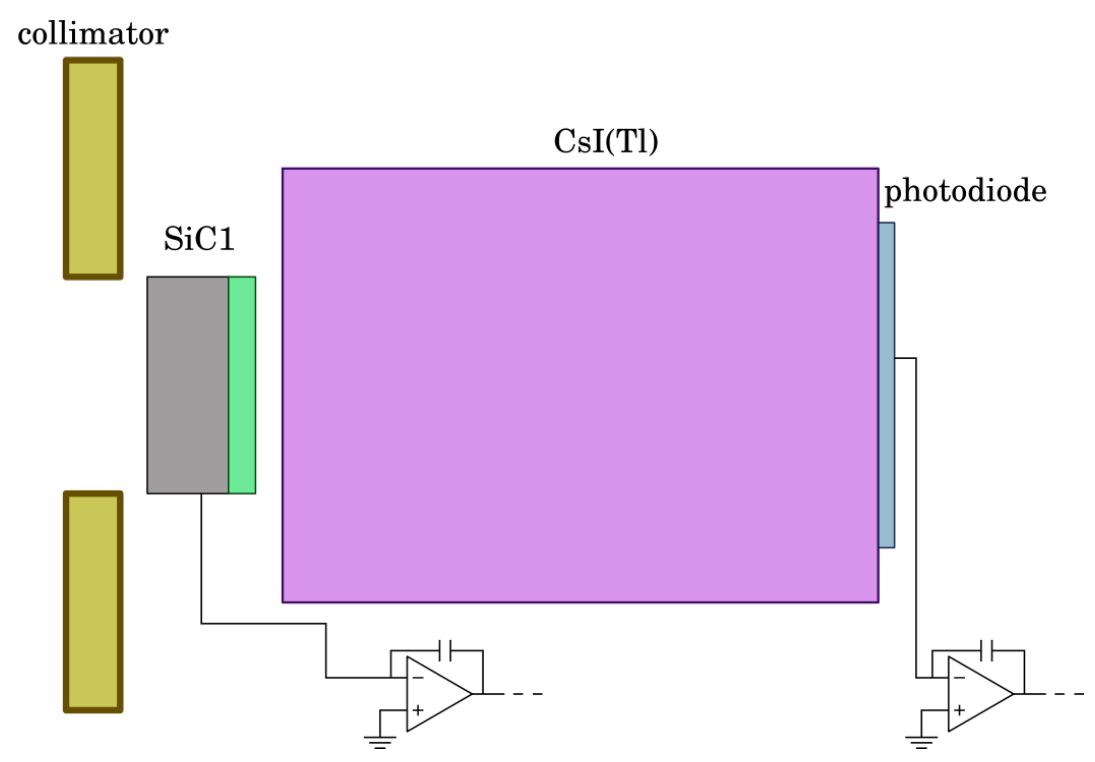

Figure 7: Sketch of telescope C. For the first element, the substrate (dark grey) and the active area (light green) are shown to scale. The picture is to scale separately for the vertical and horizontal directions.

acquired after the experiment, it is found that C-SiC1 has a depletion thickness of about $40 \mu \mathrm{m}$ at the bias voltage employed during the experiment $(150 \mathrm{~V})$. Therefore, it is not completely depleted. This could affect its calibration. The energy information coming from $\mathrm{C}-\mathrm{SiC} 1$ has not been calibrated.

A $\Delta \mathrm{E}-\mathrm{E}$ correlation obtained from $\mathrm{C}-\mathrm{SiC} 1 \mathrm{~b}$ and $\mathrm{C}-\mathrm{CsI}$ is shown in Fig. 8 in ADC units (C-SiCla gives a similar performance). The different elements are well identified in the whole range covered by the reaction products (up to $\mathrm{Z} \approx 20$ ). An expanded view is shown in the inset, demonstrating that some isotopic identification is achieved, albeit only for $\mathrm{Z} \lesssim 3$. The linearization procedure yields the PI spectrum shown in Figure9. Only fragments producing an amplitude of at least 40 a.u. have been taken into account. No isotopic separation is visible, except for the hydrogen isotopes (as evidenced by the inset) and the ${ }^{7} \mathrm{Be}-{ }^{9} \mathrm{Be}$ pair. For lithium one gets a single peak since isotopic separation is achieved only in a limited energy range.

Table 5 reports the FoM's obtained from the PI spectrum of Fig. 9. Identifi- 


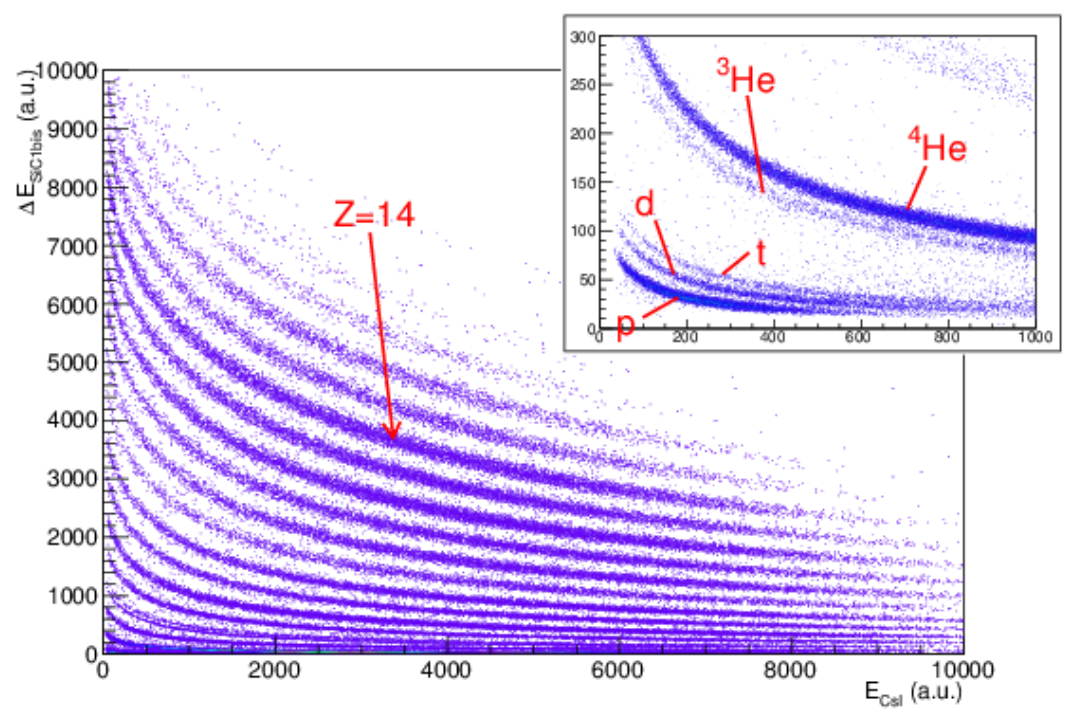

Figure 8: $\Delta \mathrm{E}$-E correlation obtained from the two-stage telescope $\mathrm{C}$ (C-SiC1b and C-CsI). The inset shows an expanded view of the $\mathrm{Z} \leq 2$ region.

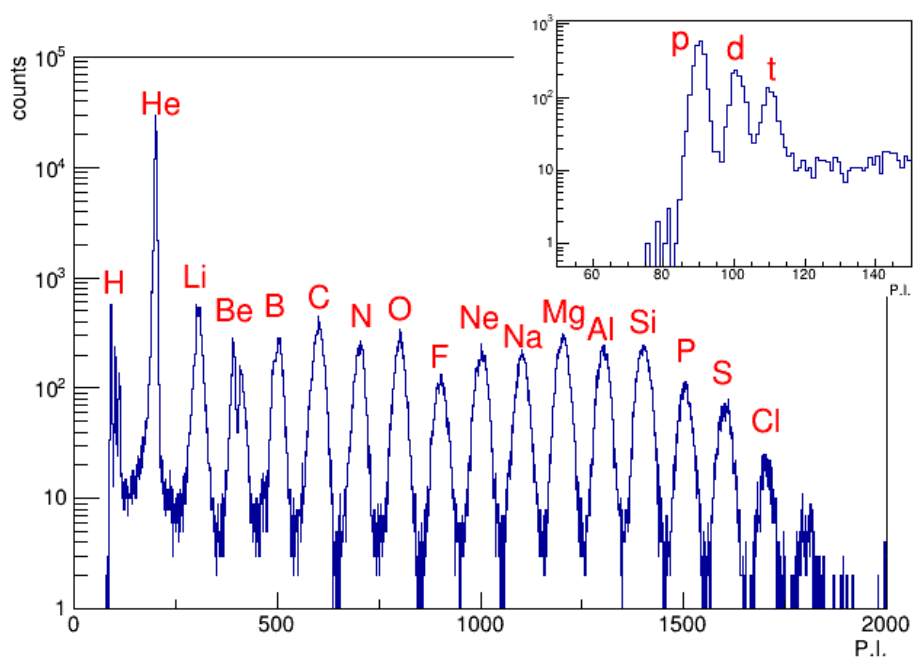

Figure 9: PI spectrum obtained from a linearization of the $\Delta \mathrm{E}-\mathrm{E}$ correlation of Fig. 8 Only fragments for which the $\Delta \mathrm{E}$ amplitude is $>40$ a.u. are included. The inset shows the PI spectrum for hydrogen isotopes (PI 100). 
Table 5: FoM values for isotope and element pairs as obtained from the $\Delta \mathrm{E}-\mathrm{E}$ correlation of C-SiC1b vs C-CsI (full energy range).

\begin{tabular}{lc||lc||lc}
\hline Isotopes & FoM & Elements & FoM & Elements & FoM \\
\hline $\mathrm{p}-\mathrm{d}$ & \multirow{2}{*}{1.4} & B-C & 1.9 & Na-Mg & 1.6 \\
$\mathrm{~d}-\mathrm{t}$ & 1.1 & $\mathrm{C}-\mathrm{N}$ & 1.9 & $\mathrm{Mg}-\mathrm{Al}$ & 1.5 \\
${ }^{7} \mathrm{Be}^{9} \mathrm{Be}$ & 0.7 & $\mathrm{~N}-\mathrm{O}$ & 1.9 & $\mathrm{Al}-\mathrm{Si}$ & 1.5 \\
& \multirow{2}{*}{} & O-F & 1.8 & $\mathrm{Si}-\mathrm{P}$ & 1.6 \\
& & F-Ne & 1.6 & $\mathrm{P}-\mathrm{S}$ & 1.5 \\
& & Ne-Na & 1.6 & S-Cl & 1.6 \\
\hline
\end{tabular}

cation is satisfactory $(\mathrm{FoM}>0.7)$ for all the elements and for hydrogen isotopes. However, one cannot exclude that a better performance could be achieved by using a completely depleted $\Delta \mathrm{E}$ detector.

\section{Identification from PSA}

This section presents a study of the PSA identification capability of the SiC detectors B-SiC1 and B-SiC1bis.

Two PS related observables have been used. The first is the rise-time of the charge preamplifier output (charge signal). The charge signal has been first filtered through a trapezoidal shaper to optimize the signal-to-noise ratio before extracting its maximum value. The rise-time has been evaluated using a digital Constant Fraction Discriminator algorithm based on cubic interpolation [29]. The second observable is the maximum value of the current signal [30]. The current signal has been obtained from the charge signal through digital differentiation, exploiting a pole-zero cancellation and interpolation algorithm based on smoothing splines 26, 31]. The smoothing spline interpolation gives a better signal-to-noise-ratio than interpolation alone. The maximum value is extracted from the interpolated signal. Nine values are calculated for each sampling period, i.e. the distance between consecutive values after interpolation is $0.8 \mathrm{~ns}$. 


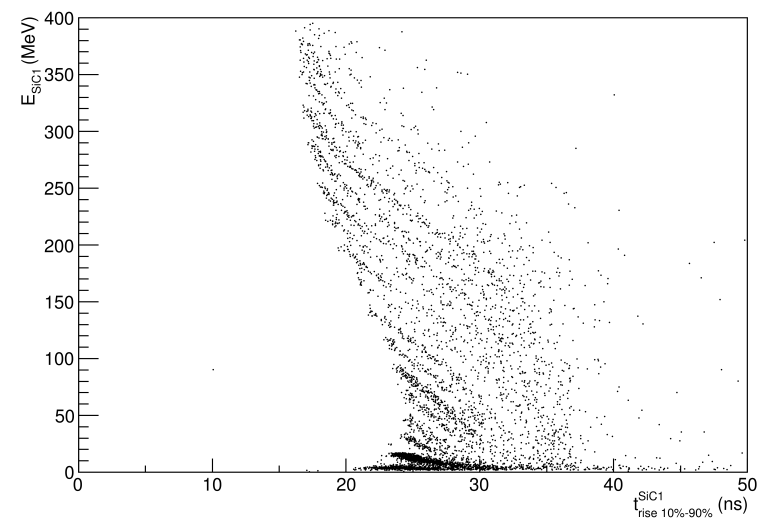

(a)

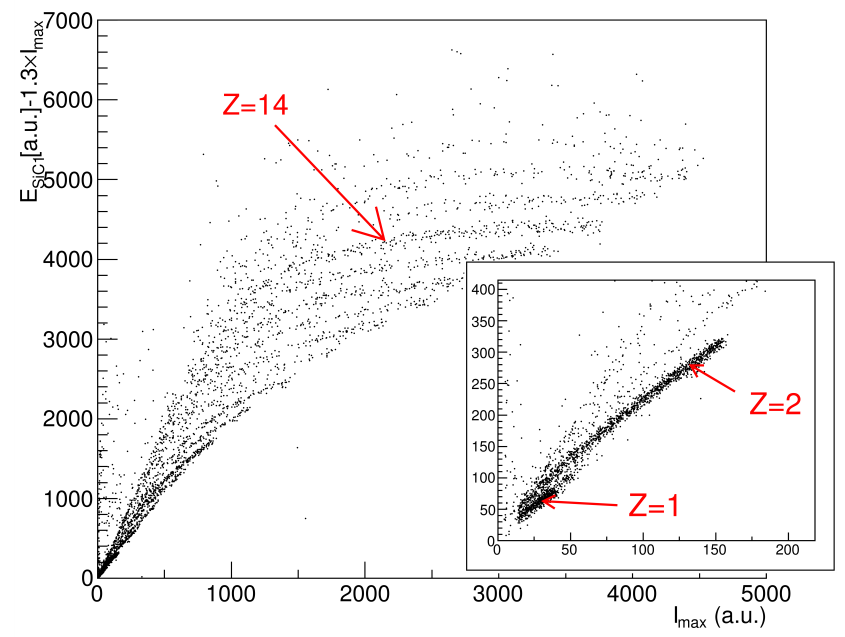

(b)

Figure 10: PS identification for fragments stopped in B-SiC1. Panel (a): Deposited energy vs Rise Time of the charge signal. Panel (b): $\mathrm{E}-1.3 \mathrm{I}_{\max }$ vs $\mathrm{I}_{\max }$; the inset shows the region of $\mathrm{Z} \leq 2$ fragments.

\section{1. $B-S i C 1$}

Figure 10 shows two PSA correlations obtained from B-SiC1. By requiring the energy measured by B-Si2 to be compatible with the noise level, only fragments stopped in $\mathrm{B}-\mathrm{SiC} 1$ are considered in this analysis. In Fig. 10a we 


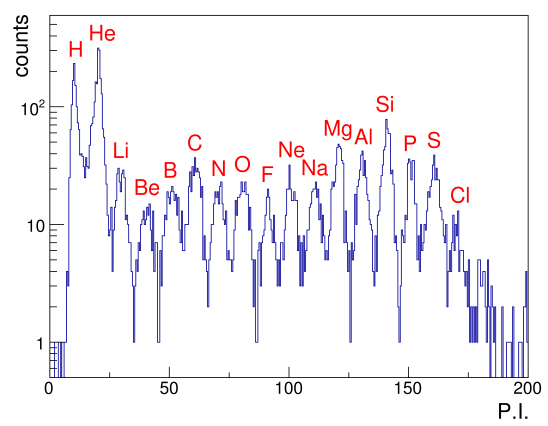

(a)

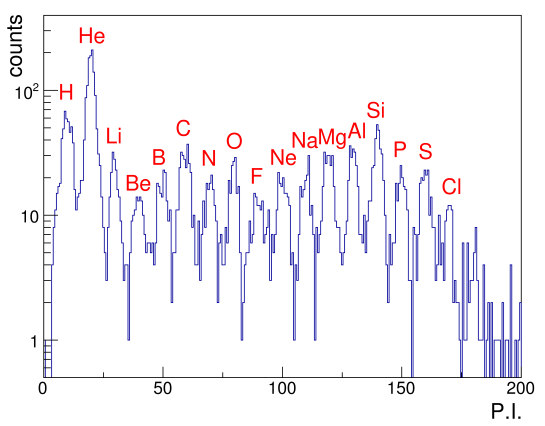

(b)

Figure 11: PS identification for fragments stopped in B-SiC1. Panel (a): PI spectrum obtained from the Energy vs Rise Time correlation of Fig. 10a Panel (b): PI spectrum obtained from the Energy vs Current Maximum correlation of Fig. 10b

Table 6: FoM values for element pairs as obtained from the PSA applied to B-SiC1, using either the rise-time of the charge signal (see Fig. 10a or the maximum of the current signal (see Fig. 10b).

\begin{tabular}{lcc||lcc}
\hline Pair & FoM (charge) & Fom (current) & Pair & FoM (charge) & Fom (current) \\
\hline H-He & 1.4 & 1.1 & Ne-Na & 0.9 & 1.0 \\
He-Li & 1.0 & 1.2 & Na-Mg & 0.9 & 1.0 \\
Li-Be & 0.9 & 1.0 & Mg-Al & 1.1 & 1.1 \\
Be-B & 0.9 & 1.0 & Al-Si & 1.1 & 1.2 \\
B-C & 0.8 & 0.9 & Si-P & 1.2 & 1.1 \\
C-N & 0.8 & 1.0 & P-S & 0.9 & 1.0 \\
N-O & 0.9 & 1.2 & S-Cl & 0.8 & 1.2 \\
O-F & 1.0 & 0.9 & Cl-Ar & 1.8 & n.a. \\
F-Ne & 0.9 & 0.8 & & & \\
\hline
\end{tabular}

report the correlation of the deposited energy, E, vs the rise-time (10\% to $90 \%$ of the maximum value) of the charge signal. In the correlation of Fig. $10 \mathrm{~b}$ the maximum of the current signal, $\mathrm{I}_{\max }$ is used for the abscissa while the ordi- 

the correlation. The ridges associated to the different elements can be clearly identified in both panels (even for the light elements, as evidenced by the inset in Fig. 10b. After linearization, the PI spectra shown in Fig. 11 are obtained. Table 6 summarizes the PSA performance of telescope B in terms of FoM for different element pairs.

The FAZIA collaboration has shown [30, 32] that the Energy vs $\mathrm{I}_{\max }$ correlation produces a better isotopic separation when PSA is applied to silicon detectors. The present measurement does not allow for a definite conclusion for the $\mathrm{SiC}$ detectors. It is not possible to recognize a consistent pattern in the data of Tab. 6. The FoM obtained from the two correlations agree within the estimated uncertainty (10\%). Poor detector quality (e.g. insufficient doping uniformity) or effects due to the employed electronics (not optimized for PSA) could cause a resolution loss, thus partly concealing the difference between the two PSA methods.

Rough estimates of the energy thresholds for element identification have been obtained from the correlation of Fig. 10a and they are reported in Tab. 7 . Their evaluation from a study of the FoM values as a function of the particle

Table 7: Energy thresholds for element identification by PSA based on the correlation "E vs rise-time of the charge signal". Values are obtained from Fig. 10a

\begin{tabular}{lc||lc}
\hline Element & Threshold $(\mathrm{MeV})$ & Element & Threshold $(\mathrm{MeV})$ \\
\hline $\mathrm{H}$ & 2 & $\mathrm{~F}$ & 70 \\
$\mathrm{He}$ & 4 & $\mathrm{Ne}$ & 90 \\
$\mathrm{Li}$ & 10 & $\mathrm{Na}$ & 100 \\
$\mathrm{Be}$ & 15 & $\mathrm{Mg}$ & 120 \\
$\mathrm{~B}$ & 20 & $\mathrm{Al}$ & 150 \\
$\mathrm{C}$ & 30 & $\mathrm{Si}$ & 170 \\
$\mathrm{~N}$ & 35 & $\mathrm{P}$ & 200 \\
$\mathrm{O}$ & 40 & $\mathrm{~S}$ & 250 \\
\hline
\end{tabular}




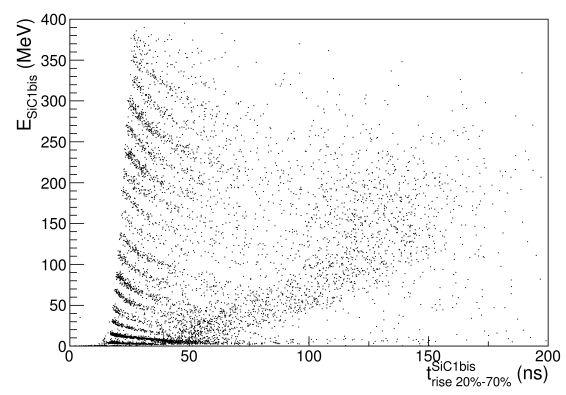

(a)

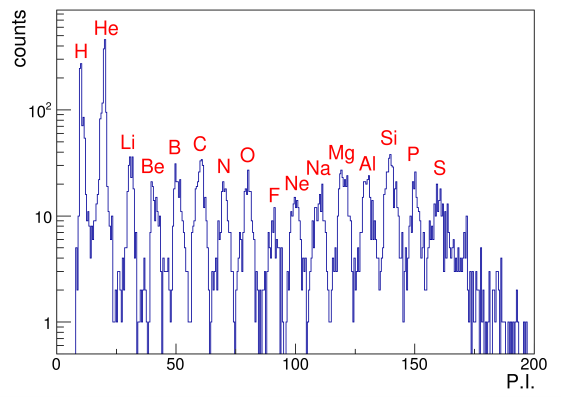

(b)

Figure 12: a) Energy vs Rise-Time (20\% to $70 \%$ ) for B-SiC1bis; b) PI spectrum as obtained from a linearization of the correlation shown in panel a).

energy (as done in [10]) is prevented by the low statistics.

\section{2. $B$-SiC1bis}

The three pads which constitute detector B-SiC1 are over-biased by a factor of six. Therefore their PSA performance could be less than optimal, assuming the same considerations apply for $\mathrm{SiC}$ as for silicon [32]. The over-bias factor for detector B-SiC1bis is much smaller $(<2)$. Figure $12 \mathrm{a}$ presents a correlation Energy vs Rise-Time. The rise-time from $20 \%$ to $70 \%$ of the maximum value of the charge signal is used, since this choice gives the best results for B-SiC1bis. The PI spectrum obtained from the correlation of Fig. 12a is presented in Fig. 12b. FoM values have been calculated for adjacent peaks and they are listed in Tab. 8. The FoM is greater than 0.7 for all the element pairs. A comparison with Tab. 6 confirms that the PSA performance of a detector biased close to the depletion voltage is better than for an over-biased detector. Figure 13 shows that even some isotopic resolution is achieved in the region of light charged particles $(\mathrm{Z} \leq 2)$. 


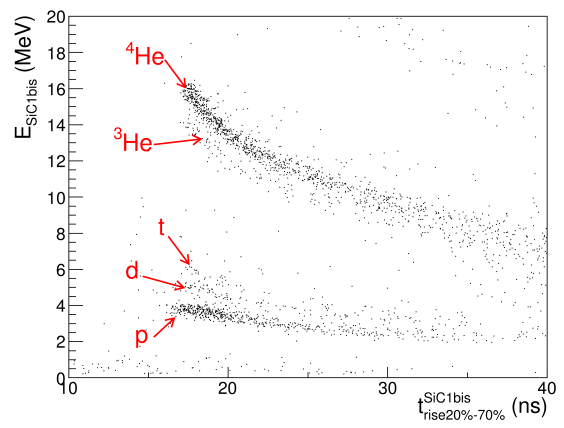

(a)

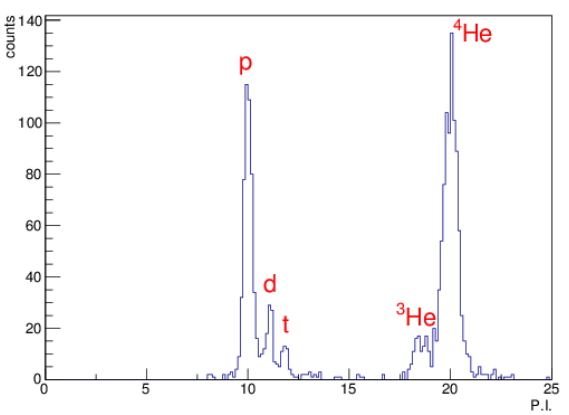

(b)

Figure 13: a) Energy vs Rise-Time (20\% to $70 \%$ ) correlation for B-SiC1bis, showing the region of light charged particles; b) PI spectrum showing $\mathrm{H}$ and He isotopes, as obtained from the correlation shown in panel a).

\section{Conclusions}

For the first time in this work, $\mathrm{SiC}$ detectors have been employed in a typical arrangement employed in nuclear physics experiment, the $\Delta \mathrm{E}-\mathrm{E}$ telescope. Three detector telescopes featuring $\mathrm{SiC}$ detectors prototypes produced by the

Table 8: FoM values for element pairs as obtained from PSA applied to B-SiC1bis using the rise-time of the charge signal.

\begin{tabular}{lc||lc}
\hline Pair & FoM & Pair & FoM \\
\hline H-He & 5.1 & F-Ne & 1.0 \\
He-Li & 3.1 & Ne-Na & 1.2 \\
Li-Be & 1.7 & Na-Mg & 1.2 \\
Be-B & 1.4 & Mg-Al & 1.2 \\
B-C & 1.3 & Al-Si & 1.3 \\
C-N & 1.3 & Si-P & 1.4 \\
N-O & 1.4 & P-S & 1.2 \\
O-F & 1.1 & & \\
\hline
\end{tabular}


SiCilia collaboration have been tested with fragments coming from the nuclear reactions induced by $\mathrm{Ca}$ beams at $40 \mathrm{AMeV}$.

Fragment identification via the $\Delta \mathrm{E}-\mathrm{E}$ technique has been studied.

For a SiC-SiC-CsI telescope employing a $13 \mu \mathrm{m}$ thick $\mathrm{SiC}$ detector as $\Delta \mathrm{E}$ stage and a $100 \mu \mathrm{m}$ thick $\mathrm{SiC}$ detector as second stage, we achieve good element identification (at least up to $\mathrm{Z}=20$ ) with low energy threshold $(\sim 1.5 \mathrm{AMeV}$ ). However, the physical limit due to the energy straggling in the first stage has not been reached yet. Further studies are needed in order to identify the factors limiting the performance.

For a SiC-Si-CsI telescope employing a $100 \mu \mathrm{m}$ thick $\mathrm{SiC}$ detector as $\Delta \mathrm{E}$ stage and a $510 \mu \mathrm{m}$ thick silicon detector as second stage, we obtain good element identification (at least up to $\mathrm{Z}=20$ ) and isotopic identification up to $\mathrm{Z}=14$.

A two-layer SiC-CsI telescope employing a $100 \mu \mathrm{m}$ thick SiC detector as $\Delta \mathrm{E}$ stage and a $10 \mathrm{~cm}$ thick $\mathrm{CsI}(\mathrm{Tl})$ scintillator as second stage provides good element identification (at least up to $\mathrm{Z}=20$ ). Isotopic identification is obtained for hydrogen isotopes and, in a limited energy interval, for fragments with $\mathrm{Z} \leq 4$.

Pulse shape analysis has been applied to the signals produced by the $100 \mu \mathrm{m}$ thick $\mathrm{SiC}$ detector, obtaining promising results. Good element identification and, for the pad biased at the lower voltage, even some isotopic identification for light charged particles has been obtained. Since poor statistics prevents a detailed study of PSA in the present work, further measurements are planned. In particular a systematic study of the PSA performance as a function of the applied bias, as done in Ref. [10, is needed, including a careful evaluation of the energy threshold for identification.

\section{Acknowledgements}

The authors would like to thank the LNS Superconducting Cyclotron staff, in particular G. Cosentino, for providing a very high-quality beam. The skillful assistance of E. Scarlini of Università di Firenze and M. Brianzi of INFN-Sezione 
help in mounting the telescopes and to the Machine Shop team of LNS-Catania for the manufacturing of the collimators. This research was funded by Istituto Nazionale di Fisica Nucleare (INFN).

\section{References}

370 [1] S. Wuenschel, K. Hagel, R. Wada, et al., NIMROD-ISiS, a versatile tool for studying the isotopic degree of freedom in heavy ion collisions, Nuclear Instruments and Methods in Physics Research Section A: Accelerators, Spectrometers, Detectors and Associated Equipment 604 (3) (2009) 578 583. doi:https://doi.org/10.1016/j.nima.2009.03.187.

URL http://www.sciencedirect.com/science/article/pii/ S016890020900446X

q [2] Pagano, E.V., Acosta, L., Auditore, L., et al., The FARCOS project -

口 status and perspective, EPJ Web of Conferences 88 (2015) 00013. doi: 10.1051/epjconf/20158800013.

n [3] B. Davin, R. de Souza, R. Yanez, et al., Lassa: a large area silicon strip array for isotopic identification of charged particles, Nuclear Instruments and Methods in Physics Research Section A: Accelerators, Spectrometers, Detectors and Associated Equipment 473 (3) (2001) 302 - 318. doi:https://doi.org/10.1016/S0168-9002(01)00295-9. URL http://wwW.sciencedirect.com/science/article/pii/ S0168900201002959

q [4] N. L. Neindre, M. Alderighi, A. Anzalone, et al., Mass and charge identification of fragments detected with the chimera Silicon-CsI(Tl) telescopes Nuclear Instruments and Methods in Physics Research Section A: Accelerators, Spectrometers, Detectors and Associated Equipment 490 (1) (2002) 251 - 262. doi:https://doi.org/10.1016/S0168-9002(02)01008-2 
URL http://www.sciencedirect.com/science/article/pii/ S0168900202010082

395 [5] M. Assi, A. Matta, B. L. Crom, et al., New methods to identify low energy ${ }^{3} \mathrm{He}$ with silicon-based detectors, Nuclear Instruments and Methods in Physics Research Section A: Accelerators, Spectrometers, Detectors and Associated Equipment 908 (2018) 250 - 255. doi:https://doi.org/10.1016/j.nima.2018.08.050.

400 uRL http://www.sciencedirect.com/science/article/pii/ S0168900218310088

口 [6] R. Bougault, G Poggi, S Barlini, et al., The fazia project in europe: 1 R\&D phase, Eur. Phys. J. A 50 (2) (2014) 47. doi:10.1140/epja/ i2014-14047-4

405 URL http://dx.doi .org/10.1140/epja/i2014-14047-4

a [7] L. Bardelli, M. Bini, G. Casini, et al., Progresses in the pulse a shape identification with silicon detectors within the fazia collaboration, Nucl. Instrum. Methods A 654 (1) (2011) 272 - 278. doi:http://dx.doi.org/10.1016/j.nima.2011.06.063

410 URL http://www.sciencedirect.com/science/article/pii/ S0168900211011879

q [8] S. Carboni, S. Barlini, L. Bardelli, et al., Particle identification using the a technique and pulse shape discrimination with the silicon detectors of the fazia project, Nucl. Instrum. Methods A 664 (1) (2012) 251 - 263.

415 doi:http://dx.doi.org/10.1016/j.nima.2011.10.061

1) URL http://wWw.sciencedirect.com/science/article/pii/ S0168900211020134

q [9] N. L. Neindre, R. Bougault, S. Barlini, et al., Comparison घ of charged particle identification using pulse shape discrimina${ }_{420}^{4}$ tion and methods between front and rear side injection in silicon detectors, Nucl. Instrum. Methods A 701 (2013) 145 - 152. 
doi:http://dx.doi.org/10.1016/j.nima.2012.11.005

URL http://www.sciencedirect.com/science/article/pii/ S0168900212012764

${ }_{425}^{0}$ 10] G. Pasquali, Pastore, G., Le Neindre, N., et al., Energy measurement and a fragment identification using digital signals from partially depleted si detectors, Eur. Phys. J. A 50 (5) (2014) 86. doi:10.1140/epja/i2014-14086-9. URL http://dx.doi.org/10.1140/epja/i2014-14086-9

[11] Cappuzzello, F., Agodi, C., Cavallaro, M., et al., The numen project: Nuclear matrix elements for neutrinoless double beta decay, Eur. Phys. J. A 54 (5) (2018) 72. doi:10.1140/epja/i2018-12509-3. URL https://doi.org/10.1140/epja/i2018-12509-3

[12] S. Barlini, S. Carboni, L. Bardelli, et al., Effects of irradiation of energetic heavy ions on digital pulse shape analysis with silicon detectors, Nuclear Instruments and Methods in Physics Research Section A: Accelerators, Spectrometers, Detectors and Associated Equipment 707 (2013) 89 - 98. doi:https://doi.org/10.1016/j.nima.2012.12.104. URL http://www.sciencedirect.com/science/article/pii/ S0168900212016609

[13] F. Negoita, M. Roth, P. Thirlof, et al., Laser driven nuclear physics at elinp, Romanian Reports in Physics 68 (Supplement) (2016) S37-S144.

[14] G. A. Cirrone, M. Carpinelli, G. Cuttone, et al., Elimed, future hadrontherapy applications of laser-accelerated beams, Nuclear Instruments and Methods in Physics Research Section A: Accelerators, Spectrometers, Detectors and Associated Equipment 730 (2013) 174 - 177, proceedings of the 9th International Conference on Radiation Effects on Semiconductor Materials Detectors and Devices. doi:https://doi.org/10.1016/j.nima.2013.05.051.

a URL http://www.sciencedirect.com/science/article/pii/


[15] S. Tudisco, F. La Via, C. Agodi, et al., Sicilia-silicon carbide detectors for

1 intense luminosity investigations and applications, Sensors 18 (7). doi: $10.3390 / \mathrm{s} 18072289$

URL http://www .mdpi.com/1424-8220/18/7/2289

[17] J. England, G. Field, T. Ophel, Z-identification of charged particles by signal risetime in silicon surface barrier detectors Nucl. Instrum. Methods A 280 (1989) 291 - 298 . doi:http: //dx.doi.org/10.1016/0168-9002(89)90920-0.

460 URL http://www.sciencedirect.com/science/article/pii/ 0168900289909200

[18] G. Pausch, W. Bohne, H. Fuchs, et al., Particle identificaa tion in solid-state detectors by exploiting pulse shape information, Nucl. Instrum. Methods A 322 (1) (1992) 43 - 52. doi:http://dx.doi.org/10.1016/0168-9002(92)90356-9.

प URL http://www.sciencedirect.com/science/article/pii/ 0168900292903569

[19] C.A.Ammerlaan., Particle identification by pulse shape discrimination in the p-i-n type semiconductor detector, Nucl. Instrum. Methods A 22 (22) (1963) 189.

[20] M. Mutterer, W. H. Trzaska, G. P. Tyurin, et al., Breakthrough in pulseshape based particle identification with silicon detectors, IEEE Transactions on Nuclear Science 47 (3) (2000) 756-759. doi:10.1109/23.856510

[21] G. Pausch, H. . Ortlepp, W. Bohne, et al., Identification of light charged 475 particles and heavy ions in silicon detectors by means of pulse-shape discrimination, IEEE Transactions on Nuclear Science 43 (3) (1996) 10971101. doi:10.1109/23.506644. 
[22] W. Choyke, G. Pensl, Physical properties of sic, MRS Bulletin 22 (3) (1997) 2529. doi:10.1557/S0883769400032723.

480

485

[24] [link]

URL https://www.fbk.eu

[25] M. Bruno, F. Gramegna, T. Marchi, et al., Garfield + RCo digital up-

1 grade: a modern set-up for mass and charge identication of heavy ion 490

口

[26] P. Ottanelli, Real-time extraction of energy and shape parameters of

URL magistrale.pdf

[27] R. Winyard, J. Lutkin, G. McBeth, Pulse shape discrimination in inorganic and organic scintillators. i, Nucl. Instrum. Methods 95 (1) (1971) 141 153. doi:http://dx.doi.org/10.1016/0029-554X(71)90054-1.

口 URL http://www.sciencedirect.com/science/article/pii/ $0029554 \times 71900541$

[28] Q. Yang, D. O'Connor, Z. Wang, Empirical formulae for energy loss straggling of ions in matter Nuclear Instruments and 
[31] T. Kurahashi, H. Takahashi, M. Nakazawa, Radiation digital signal processing using smoothing spline, Nucl. Instrum. Methods A 422 (1-3) (1999)

[32] G. Pastore, D. Gruyer, P. Ottanelli, et al., Isotopic identification using a pulse shape analysis of current signals from silicon detectors: Recent

[30] S. Barlini, R. Bougault, P. Laborie, et al., New digital techniques applied

1. to $\mathrm{a}$ and $\mathrm{z}$ identification using pulse shape discrimination of silicon detector current signals, Nucl. Instrum. Methods A 600 (3) (2009) 644 650. doi:http://dx.doi.org/10.1016/j.nima.2008.12.200

URL http://www.sciencedirect.com/science/article/pii/ S0168900209000023

385 - 387. doi:http://dx.doi.org/10.1016/S0168-9002(98)00988-7.

URL http://www.sciencedirect.com/science/article/pii/ S0168900298009887

results from the \{FAZIA\} collaboration, Nucl. Instr. and Meth. A 860

Materials and Atoms 61 (2) (1991) 149 - 155 doi:https:

//doi.org/10.1016/0168-583X (91)95454-L

URL http://www.sciencedirect.com/science/article/pii/

$0168583 X 9195454 \mathrm{~L}$ (2017) 42 - 50. doi:https://doi.org/10.1016/j.nima.2017.01.048.

a URL http://www.sciencedirect.com/science/article/pii/ S0168900217301092 